\title{
Correlation between the Indexes of Insulin Resistance and Renal Function in the Presence of Hypertension
}

\author{
Hiroaki Matsunaga Kazuhiko Kotani Nobuyuki Taniguchi \\ Department of Clinical Laboratory Medicine, Jichi Medical University, Shimotsuke City, Japan
}

\section{Key Words}

Insulin sensitivity · Homeostasis model assessment •

Glomerular filtration rate $\cdot$ Chronic kidney disease

\begin{abstract}
Background/Aims: It is important to establish the association between insulin resistance and renal function; however, the reported associations differ across studies. Different underlying pathophysiologies of the studied populations may affect the associations. There have been no reports on the relationship between insulin resistance and renal function in the presence of hypertension (HT). We investigated the correlation between a homeostasis model assessment of insulin resistance (HOMA-IR) and the estimated glomerular filtration rate (eGFR) in subjects with and without HT. Methods: The study included 214 individuals (mean age: 65.6 years) who were nonmedicated and cardiovascular disease-free. Clinical variables, including blood pressure (BP), creatinine, glucose and lipid panels, were measured. Results: The HT group showed significantly higher levels of systolic/diastolic $\mathrm{BP}$ than the non-HT group. A multiple linear regression analysis revealed that the eGFR in the non-HT group was independently, significantly and inversely correlated with HOMA$I R$, while the eGFR in the HT group was independently, significantly and inversely correlated with systolic BP, but not with HOMA-IR. Conclusions: A clearer correlation between HOMA-IR and eGFR was observed in the non-HT group than
\end{abstract}

the HT group, suggesting that HT may attenuate the direct correlation between the insulin resistance and renal function indices.

Copyright $\odot 2010$ S. Karger AG, Basel

\section{Introduction}

Insulin resistance (IR) is an underlying pathology of cardiometabolic disorders, such as hypertension (HT), which leads to damage of various organs including the kidney $[1,2]$. Chronic kidney disease (CKD) is a public health concern because the incidence is increasing [3]. Establishing the association between IR and CKD will be useful for the prevention of CKD at earlier stages.

Conflicting results have been presented on the association between the indices of IR and CKD traits. Some studies have reported significant inverse relationships between a homeostasis model assessment of insulin resistance (HOMA-IR, an index of IR) and the estimated glomerular filtration rate (eGFR, an index of renal function)

K.K. and N.T. assisted in study design and data interpretation. K.K. and H.M. assisted in data collection and manuscript preparation. All authors reviewed the manuscript and approved the final version submitted for publication.

\section{KARGER \\ Fax +41613061234 E-Mail karger@karger.ch} www.karger.com

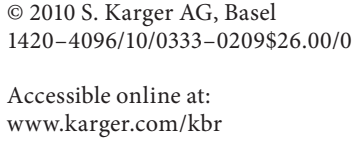
www.karger.com/kbr

Kazuhiko Kotani, MD, PhD

Department of Clinical Laboratory Medicine

Jichi Medical University

3311-1 Yakushiji, Shimotsuke-City, Tochigi, 329-0498 (Japan)

Tel. +81 28558 7386, Fax +81 28544 9947, E-Mail kazukotani@jichi.ac.jp 
in type-1 diabetic patients with mild nephropathy [4] and in non-diabetic men [5], as well as between HOMA-IR and inulin clearance (the gold standard of GFR) in obese type-2 diabetic patients with renal insufficiency [6]. On the other hand, others have reported no significant relationships between HOMA-IR and eGFR in non-diabetic patients with CKD [7] or obese non-diabetic patients [8], or between HOMA-IR and urinary albumin excretion rate in type-1 diabetic patients with mild nephropathy [9]. One study has also demonstrated that visceral adiposity (an IR-related manifestation), not HOMA-IR, is inversely associated with the eGFR in obese subjects [10]. An additional report has documented that waist circumference and metabolic syndrome (IR-related manifestations) are associated with micro-albuminuria, not eGFR, in type-2 diabetic patients [11].

These discrepancies seem largely due to the differences in such characteristics of the study population as the absence/presence of obesity and diabetes or the stage of CKD. Because renal function appears to be modulated by the states of obesity and diabetes, it is crucial to determine the situation in which the relationship between IR and renal function is most critical. While hypertensive states may also be associated with both IR and renal function, there are no reports that have examined the association of both indexes in the presence of HT. The present study therefore investigated the correlation between HOMA-IR and eGFR in the subjects with or without HT.

\section{Subjects and Methods}

A total of 214 nonmedicated participants (males/females $=$ $80 / 134$, mean age $=65.6$ years, range $=30-80$ ) were recruited from health check-ups. The eligible subjects had no severe CKD (defined as an eGFR level $<50 \mathrm{ml} / \mathrm{min} / 1.73 \mathrm{~m}^{2}$ in this study [12]) or diabetes mellitus (defined as $\leq 7.0 \mathrm{mmol} / \mathrm{l}$ of fasting plasma glucose [13]; HOMA-IR is not applicable for diabetics), as well as no apparent histories of cardiovascular, thyroid, hematological or liver diseases. The study population was composed of the HT group $(n=101)$ and the non-HT group $(n=113$; table 1$)$. The study was approved by the Jichi Medical University Ethics Committee, and all subjects gave their informed consent.

Current smoking habits were self-reported. In addition to body mass index (BMI), fasting serum total cholesterol, triglyceride, high-density lipoprotein cholesterol, creatinine and plasma glucose levels were measured by standard enzymatic methods. The plasma insulin was measured using an ELISA method (Tosoh Co. Ltd., Tokyo, Japan). Systolic blood pressure (SBP) and diastolic blood pressure (DBP) were measured in the seated subject's right-arm with a mercury sphygmomanometer after $5 \mathrm{~min}$ of rest. $\mathrm{HT}$ was diagnosed in subjects with $\geq 140 \mathrm{~mm} \mathrm{Hg}$ of SBP and/or $\geq 90 \mathrm{~mm} \mathrm{Hg}$ of DBP [14]. HOMA-IR was calculated using the fol- lowing formula: (fasting plasma insulin $\times$ plasma glucose) $/ 22.5$ [15]. The eGFR was calculated using the following formula (the Modification of Diet in Renal Disease Study Group equation): $0.741 \times 175 \times$ serum creatinine $\mathrm{s}^{-1.154} \times$ age $^{-0.203}(\times 0.742$ if female) [12].

The data were expressed as the mean \pm standard deviation (SD) or the median plus interquartile range. The data between the groups were compared using a t test or $\chi^{2}$ test. A simple and multivariate-adjusted linear regression model was utilized to observe the correlation between eGFR and HOMA-IR. In a multivariateadjusted analysis, all the measured variables were used. Because of a close correlation between glucose and HOMA-IR, these variables were not simultaneously entered in the multivariate-adjusted analysis model in this study. The triglyceride, HOMA-IR and eGFR data were log-transformed for all the analyses because of their skewed distributions. A p value of $\leq 0.05$ was considered to be statistically significant.

\section{Results}

The clinical characteristics of the HT and non-HT groups are listed in table 1 . The HT group showed significantly higher levels of BMI, SBP and DBP than the non-HT group. The HOMA-IR and eGFR levels were similar between these groups.

In the simple linear regression analysis, the eGFR was inversely and significantly correlated with HOMA-IR $(\mathrm{r}=-0.196, \mathrm{p}=0.038)$ in the non-HT group, but the eGFR showed an inverse correlation with HOMA-IR at a nonsignificant level $(\mathrm{r}=-0.182, \mathrm{p}=0.070)$ in the non-HT group. The results of the multivariate-adjustments are listed in table 2. The eGFR in the non-HT group was independently, significantly and inversely correlated with HOMA-IR, along with age. In the HT group, the eGFR was independently, significantly and inversely correlated only with SBP, and a weak correlation between the eGFR and HOMA-IR was observed.

\section{Discussion}

The present study found a significant and inverse correlation between HOMA-IR and eGFR in the non-HT group, but not in the HT group, suggesting that HT can affect the association between IR and renal function. This may be partly accounted for by the fact that HT displays glomerular hyperfiltration (via the sympathetic nervous system, angiotensin II and nitric oxide pathway) [16, 17], thus indicating that the modified (probably compensatory) levels of eGFR in such a situation may alter the direct correlation between HOMA-IR and eGFR. 
Table 1. Clinical characteristics between groups of subjects with and without hypertension

\begin{tabular}{lccc}
\hline Group variables & Non-hypertension $(\mathrm{n}=113)$ & Hypertension $(\mathrm{n}=101)$ & $\mathrm{p}$ \\
\hline Age, years & $65.7 \pm 10.5$ & $65.5 \pm 8.9$ & 0.846 \\
Males/females & $47 / 66$ & $33 / 68$ & 0.178 \\
Current smoker & 18 & 10 & 0.192 \\
Body mass index & $23.6 \pm 2.8$ & $24.6 \pm 3.1$ & $0.014^{*}$ \\
Systolic blood pressure, $\mathrm{mm} \mathrm{Hg}$ & $124.1 \pm 10.7$ & $153.9 \pm 12.8$ & $<0.0001^{* *}$ \\
Diastolic blood pressure, mm Hg & $70.9 \pm 8.0$ & $84.2 \pm 10.8$ & $<0.0001^{* *}$ \\
Total cholesterol, mmol/l & $4.87 \pm 0.94$ & $4.91 \pm 0.91$ & 0.746 \\
Triglyceride, mmol/l & $0.94(0.75-1.34)$ & $1.10(0.88-1.39)$ & 0.117 \\
HDL-cholesterol, mmol/l & $1.43 \pm 0.39$ & $1.39 \pm 0.31$ & 0.458 \\
HOMA-IR & $1.30(0.80-2.12)$ & $1.39(0.88-2.18)$ & 0.954 \\
$\quad$ Plasma glucose, $\mathrm{mmol} / \mathrm{l}$ & $5.17 \pm 0.68$ & $5.14 \pm 0.62$ & 0.752 \\
$\quad$ Plasma insulin, $\mu \mathrm{U} / \mathrm{ml}$ & $6.0(3.6-8.7)$ & $6.3(3.9-9.4)$ & 0.867 \\
eGFR, ml/min/1.73 m ${ }^{2}$ & $66.0(59.0-77.3)$ & $67.0(57.1-75.5)$ & 0.397 \\
\hline
\end{tabular}

Values are expressed as the mean \pm SD in parametrically distributed variables or median (interquartile range) in nonparametrically distributed variables. The triglyceride, HOMA-IR, insulin and eGFR data were analyzed after log-transformation because of their skewed distributions. Significance level (by t test): ${ }^{*} \mathrm{p} \leq 0.05$; ** $\mathrm{p} \leq 0.01$. HDL $=$ High-density lipoprotein.

Table 2. Multivariate-adjusted correlations of each variable on eGFR $\left(\mathrm{ml} / \mathrm{min} / 1.73 \mathrm{~m}^{2}\right)$ in the respective groups of subjects with and without hypertension

\begin{tabular}{llllll}
\hline \multirow{2}{*}{ Group variables } & \multicolumn{2}{l}{ Non-hypertension } & & \multicolumn{2}{l}{ Hypertension } \\
\cline { 2 - 3 } & $\beta$-coefficient & $\mathrm{p}$ & & $\beta$-coefficient & $\mathrm{p}$ \\
\hline Age, year & -0.352 & $0.001^{* *}$ & & -0.160 & 0.129 \\
Gender, male & -0.028 & 0.794 & & 0.023 & 0.841 \\
Current smoking & -0.178 & 0.109 & & 0.005 & 0.961 \\
Body mass index & -0.183 & 0.061 & & -0.018 & 0.872 \\
Systolic blood pressure, mm Hg & -0.020 & 0.987 & & -0.270 & $0.019^{*}$ \\
Diastolic blood pressure, mm Hg & -0.137 & 0.188 & & 0.169 & 0.162 \\
Total cholesterol, mmol/l & -0.190 & 0.120 & & 0.040 & 0.750 \\
Triglyceride, mmol/l & 0.198 & 0.091 & & -0.122 & 0.306 \\
HDL-cholesterol, mmol/l & 0.069 & 0.559 & & 0.148 & 0.228 \\
HOMA-IR & -0.211 & $0.050^{*}$ & & -0.094 & 0.409 \\
\hline
\end{tabular}

The triglyceride, HOMA-IR and eGFR data were analyzed after log-transformation because of their skewed distributions. Significance level (by multiple linear regression model analysis): ${ }^{*} \mathrm{p} \leq 0.05 ;{ }^{* *} \mathrm{p} \leq 0.01$. HDL $=$ High-density lipoprotein.
Our study had some potential limitations. The study was performed with a relatively small sample size. Because of the cross-sectional study design, we have not precisely defined any cause-and-effect relationship in the results. Prospective studies with larger sample sizes will be necessary. Furthermore, the following background should be considered: CKD is a very heterogeneous entity regardless of the causes, and the eGFR is not always an accurate estimate of true GFR (while the eGFR is more accurate than serum creatinine or the Cockcroft-Gault formula $[18,19])$. In addition, measurement of micro-albuminuria was not available for our study population. Although the single usage of eGFR to define CKD, as in our present study, has been a common approach in many epidemiological studies $[5,8,10]$, we acknowledge that this is a weakness in describing CKD in comparison with 
the other cases, where micro-albuminuria is included in the assessment of renal function. Micro-albuminuria is prevalent in diabetic and/or hypertensive patients [20, 21]. Because our study population excluded subjects with overt diabetes mellitus (only subjects with impaired glucose tolerance, impaired fasting glucose and diabetes diagnosed by the 2-hour plasma glucose concentration from an oral glucose tolerance test could not be completely excluded), there was a possibility that the measurement of micro-albuminuria was convenient for describing CKD in detail. The inclusion of micro-albuminuria measurements in such study populations as ours should be performed in the future.
In summary, the correlation between HOMA-IR and eGFR can be more clearly observed in the non-HT group than in the HT group. The presence of HT may attenuate the direct correlation between the indexes of insulin resistance and renal function. Further studies will confirm our prediction and clarify the causality of these factors.

\section{Acknowledgements}

This study was supported in part by a Grant-in-Aid for Scientific Research from the Ministry of Education, Culture, Sports, Science and Technology of Japan (K.K.), and the Foundation for the Development of the Community, Japan.

\section{References}

1 Whelton PK, Perneger TV, He J, Klag MJ: The role of blood pressure as a risk factor for renal disease: a review of the epidemiologic evidence. J Hum Hypertens 1996;10:683689.

$\checkmark 2$ Bulló M, Casas-Agustench P, Amigó-Correig P, Aranceta J, Salas-Salvadó J: Inflammation, obesity and comorbidities: the role of diet. Public Health Nutr 2007;10:11641172.

- 3 Sarnak MJ, Levey AS, Schoolwerth AC, Coresh J, Culleton B, Hamm LL, McCullough PA, Kasiske BL, Kelepouris E, Klag MJ, Parfrey P, Pfeffer M, Raij L, Spinosa DJ, Wilson PW: Kidney disease as a risk factor for development of cardiovascular disease: a statement from the American Heart Association Councils on Kidney in Cardiovascular Disease, High Blood Pressure Research, Clinical Cardiology, and Epidemiology and Prevention. Circulation 2003; 108:21542169.

-4 Svensson M, Yu ZW, Eriksson JW: A small reduction in glomerular filtration is accompanied by insulin resistance in type 1 diabetes patients with diabetic nephropathy. Eur J Clin Invest 2002;32:100-109.

5 Onat A, Hergenc G, Uyarel H, Ozhan H, Esen AM, Karabulut A, Albayrak S, Can G, Keles I: Association between mild renal dysfunction and insulin resistance or metabolic syndrome in a random nondiabetic population sample. Kidney Blood Press Res 2007; 30:88-96.

- 6 Koch M, Beien A, Fussha Ller A, Zitta S, Haastert B, Trapp R: Impact of age, body mass index insulin resistance and proteinuria on the kidney function in obese patients with Type 2 diabetes and renal insufficiency. Clin Nephrol 2008;69:10-17.
7 Satirapoj B, Supasyndh O, Boonyavarakul A, Luesutthiviboon L, Choovichian P: The correlation of insulin resistance and renal function in non diabetic chronic kidney disease patients. J Med Assoc Thai 2005;88:S97S104.

8 Gatti A, Morini E, De Cosmo SD, Maiani F, Mandosi E, Fallarino M, Morano S, Trischitta V: Metabolic syndrome is not a risk factor for kidney dysfunction in obese non-diabetic subjects. Obesity 2008;16:899-901.

-9 Svensson M, Eriksson JW: No direct link between albumin excretion rate and insulin resistance - a study in type 1 diabetes patients with mild nephropathy. Horm Metab Res 2002;34:254-259.

10 Lee JW, Bae U, Lee DC, Lee HR, Shim JY, Linton JA: Renal manifestation and visceral adiposity in apparently healthy korean women. Kidney Blood Press Res 2008;31:416420.

- 11 Hanai K, Babazono T, Iwamoto Y: Renal manifestation of metabolic syndrome in type 2 diabetes. Diabetes Res Clin Pract 2008;79:318-324.

12 Imai E, Horio M, Nitta K, Yamagata K, Iseki K, Hara S, Ura N, Kiyohara Y, Hirakata $\mathrm{H}$, Watanabe T, Moriyama T, Ando Y, Inaguma D, Narita I, Iso H, Wakai K, Yasuda Y, Tsukamoto Y, Ito S, Makino H, Hishida A, Matsuo S: Estimation of glomerular filtration rate by the MDRD study equation modified for Japanese patients with chronic kidney disease. Clin Exp Nephrol 2007;11:41-50.

13 Expert Committee on the Diagnosis and Classification of Diabetes Mellitus (American Diabetes Association): Follow-up report on the diagnosis of diabetes mellitus. Diabetes Care 2003;26:3160-3167.
14 Chobanian AV, Bakris GL, Black HR, Cushman WC, Green LA, Izzo JL Jr, Jones DW, Materson BJ, Oparil S, Wright JT Jr, Roccella EJ: The seventh report of the Joint National Committee on Prevention, Detection, Evaluation and Treatment of High Blood Pressure. Hypertension 2003;42:1206-1252.

15 Matthews DR, Hosker JP, Rudenski AS, Naylor BA, Treacher DF, Turner RC: Homeostasis model assessment: insulin resistance and beta-cell function from fasting plasma glucose and insulin concentrations in man. Diabetologia 1985;28:412-419.

16 Schmieder RE, Veelken R, Schobel H, Dominiak P, Mann JF, Luft FC: Glomerular hyperfiltration during sympathetic nervous system activation in early essential hypertension. J Am Soc Nephrol 1997;8:893-900.

-17 Tomaszewski M, Charchar FJ, Maric C, McClure J, Crawford L, Grzeszczak W, Sattar N, Zukowska-Szczechowska E, Dominiczak AF: Glomerular hyperfiltration: a new marker of metabolic risk. Kidney Int 2007; 71:816-821.

-18 Froissart M, Rossert J, Jacquot C, Paillard M, Houillier P: Predictive performance of the modification of diet in renal disease and Cockcroft-Gault equations for estimating renal function. J Am Soc Nephrol 2005;16: 763-773.

19 Imai E, Horio M, Nitta K, Yamagata K, Iseki K, Tsukamoto Y, Ito S, Makino H, Hishida A, Matsuo S: Modification of the Modification of Diet in Renal Disease (MDRD) Study equation for Japan. Am J Kidney Dis 2007; 50:927-937.

20 Stehouwer CD, Donker AJ: Clinical usefulness of measurement of urinary albumin excretion in diabetes mellitus. Neth J Med 1993;42:175-186.

21 Kalaitzidis R, Bakris G: Pathogenesis and treatment of microalbuminuria in patients with diabetes: the road ahead. J Clin Hypertens 2009;11:636-643. 